\title{
Design of a Three-Dimensional Hand/Forearm Model to Apply Computational Fluid Dynamics
}

\author{
Daniel Almeida Marinho ${ }^{1,2^{*}}$, Victor Machado Reis ${ }^{2,3}$, João Paulo Vilas-Boas ${ }^{4}$, Francisco \\ Bessone Alves $^{5}$, Leandro Machado ${ }^{4}$, Abel Ilah Rouboa ${ }^{3,6}$ and António José Silva ${ }^{2,3}$ \\ ${ }^{1}$ Departamento de Ciências do Desporto; Universidade da Beira Interior; Rua Marquês d'Ávila e Bolama; 6201- \\ 001; Covilhã -Portugal. ${ }^{2}$ Centro de Investigação de Desporto, Saúde e Desenvolvimento Humano; Apartado 1013; \\ 5001-801; Vila Real - Portugal. ${ }^{3}$ Departamento de Ciências do Desporto; Universidade de Trás-os-Montes and Alto \\ Douro; Apartado 1013; 5001-801 Vila Real - Portugal. ${ }^{4}$ Universidade do Porto; Faculdade de Desporto; Rua Dr. \\ Plácido Costa; 91; 4200-450; Porto - Portugal. ${ }^{5}$ Faculdade de Motricidade Humana; Universidade Técnica de \\ Lisboa; Estrada da Costa; 1495-688; Cruz Quebrada - Portugal. ${ }^{6}$ Centro de Investigação Tecnologias Agro- \\ Ambientais e Biológicas; Apartado 1013; 5001-801; Vila Real-Portugal
}

\begin{abstract}
The purpose of this study was to develop a three-dimensional digital model of a human hand and forearm to apply Computational Fluid Dynamics to propulsion analysis in swimming. Computer tomography scans of the hand and forearm of an Olympic swimmer were applied. The data were converted, using image processing techniques, into relevant coordinate input, which could be used in Computational Fluid Dynamics software. From that analysis, it was possible to verify an almost perfect agreement between the true human segment and the digital model. This technique could be used as a means to overcome the difficulties in developing a true three-dimensional model of a specific segment of the human body. Additionally, it could be used to improve the use of Computational Fluid Dynamics generally in sports and specifically in swimming studies, decreasing the gap between the experimental and the computational data.
\end{abstract}

Key words: Finite element modelling, Image processing, Computational fluid dynamics, Biomechanics, Swimming

\section{INTRODUCTION}

The finite element method is currently one of the best established numerical tools in the field of biomechanical engineering and has been used in the computational analysis of the fluid flow around human structures. In the sports scope, it has been used in the study of the propulsive forces produced by the hand and forearm in human swimming. Despite the increasing amount of high quality research, a common weakness still remains. Practically all the models have been developed based on approximate analytical representations of the human structures and their geometrical accuracy has never been discussed. This approach has been commonly adopted, for example, to reduce the computational cost of memory requirements (Aritan et al., 1997). However, one of the main reasons for such limitations is the difficulty to design a true digital model of the

*Author for correspondence: dmarinho@ubi.pt 
human limbs.

In most cases, the authors used two-dimensional models (Bixler and Schloder, 1996; Silva et al., 2005; Rouboa et al., 2006) and when threedimensional models were used, these were very simple and reductive representations of the human limbs (Gardano and Dabnichki, 2006). These differences between the true and computed models could lead to less accurate numerical results (Candalai and Reddy, 1992). In an experimental simulation of the effect of the ischial tuberosity's geometry on the shear and compressive stress in buttock tissue, Candalai and Reddy (1992) showed that the influence of the geometry on the stress magnitude could be significant. In a numerical simulation of this experimental work, a possible variation of more than $60 \%$ was found in the shear stress.

Magnetic resonance imaging and computer tomography scans seem to be a good approach for designing the true human models. However, the use of these kinds of scans is not a straightforward task and requires the implementation of image processing and other numerical techniques, such as the conversion into relevant coordinate input. It should be noted that mesh generation, the first step in finite element modeling, is a tough procedure, especially when solving the three-dimensional problems (Aritan et al., 1997). Ideally, a mesh should allow modifications, usually by changing some predefined parameters and it should be based on directly obtained anatomical data. Thus, it is important to use the magnetic resonance imaging or the computer tomography data to provide geometrical input for generation or modification of finite element models. Most of the wellestablished finite element software packages provide special tools for parametric modification of an existing mesh. These tools help the user to reduce the time and to increase the accuracy and reliability of model modifications.

Therefore, the aim of the present study was to develop a true three-dimensional model of the human hand and forearm, through the transformation of computer tomography scans into input data to apply Computational Fluid Dynamics to the propulsion analysis in swimming.

\section{METHODS}

\section{Computational Fluid Dynamics}

Computational Fluid Dynamics methodology consists of a mathematical model applied to the fluid flow in a given domain. This domain replaces the Navier-Stokes equations with discretized algebraic expressions that can be solved by iterative calculations. This domain consists of a three-dimensional grid or mesh of cells that simulates the fluid flow around structures. The fluid mechanical properties, the flow characteristics along the outside grid boundaries and the mathematical relationship to account the turbulence were also considered:

$$
\begin{aligned}
& \operatorname{div} \mathrm{V}=0 \\
& \frac{\partial \mathrm{V}}{\partial \mathrm{t}} \pm \mathrm{V} \cdot \nabla \mathrm{V}+\nabla \mathrm{p} \pm \nabla\left(\mathrm{v}+\mathrm{c}_{\mu} \frac{\mathrm{k}^{2}}{\varepsilon}\right)\left(\nabla \mathrm{V}+\nabla \mathrm{V}^{\mathrm{t}}\right)=0
\end{aligned}
$$

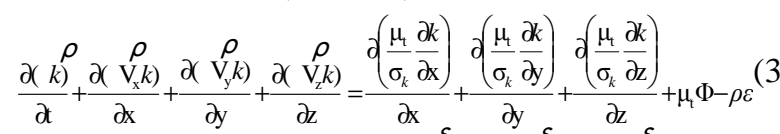

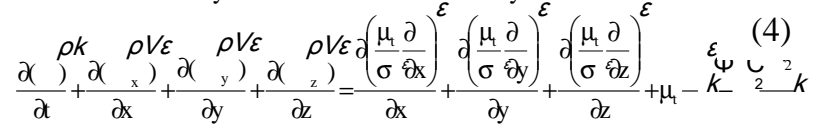

Where $k$ is the turbulent kinetic energy and $\varepsilon$ is the turbulent kinetic energy dissipation ratio. $V_{x}, \mathrm{~V}_{\mathrm{y}}$ and $V_{z}$ represent the $\mathrm{x}, \mathrm{y}$ and $\mathrm{z}$ components of the velocity $V . \mu_{\mathrm{t}}$ is the turbulent viscosity and $\rho$ represents the fluid density. $v$ is the kinematic viscosity, $\Phi$ is the pressure strain, $C_{2}, C_{\mu}, \sigma_{\varepsilon}$ and $\sigma_{k}$ are model constants, $1.92,0.09,1.30$ and 1.00 , respectively.

In order to create the three-dimensional digital model, computer tomography scans of a hand and forearm segments of an Olympic swimmer were applied. With these data, the values were converted into a format that could be read in Gambit, Fluent ${ }^{\circledR}$ pre-processor. Fluent ${ }^{\circledR}$ software was used to simulate the fluid flow around structures, allowing the analysis of values of pressure and speed around (i.e. the hand and forearm of a swimmer). With these values, force components through the integration of pressures on the hand/forearm surfaces were calculated, using a realistic model of these human segments, thus decreasing the gap between the experimental and the computational data.

The numerical method used by Fluent ${ }^{\circledR}$ is based on the finite volume approach. The solutions of the governing system equations are given in each square element of the discretized whole domain. In order to solve the linear system, Fluent ${ }^{\circledR}$ code adopts an Algebraic Multi-Grid (AMG) solver. Velocity components, pressure, turbulent kinetic energy and turbulent kinetic energy dissipation 
ratio are degrees of freedom (DOF) for each element.

\section{Computer tomography scans}

Eighteen cross-sectional scans of the right arm (hand and forearm) were obtained using a Toshiba $^{\circledR}$ Aquilion 4 computer tomography scanner. Computer tomography scans were obtained with configuration of V2.04 ER001. A 2 $\mathrm{mm}$ slice thickness with a space of $1 \mathrm{~mm}$ was used. The subject was an Olympic level swimmer, who participated in the 2004 Olympic Games in Athens. The subject was lying with his right arm extended upwards and fully pronated. The thumb was adducted and the wrist was in a neutral position (Fig. 1). This protocol has been approved by the appropriate ethical committee of the institution in which it was performed, and the subject consented to participate in this work.
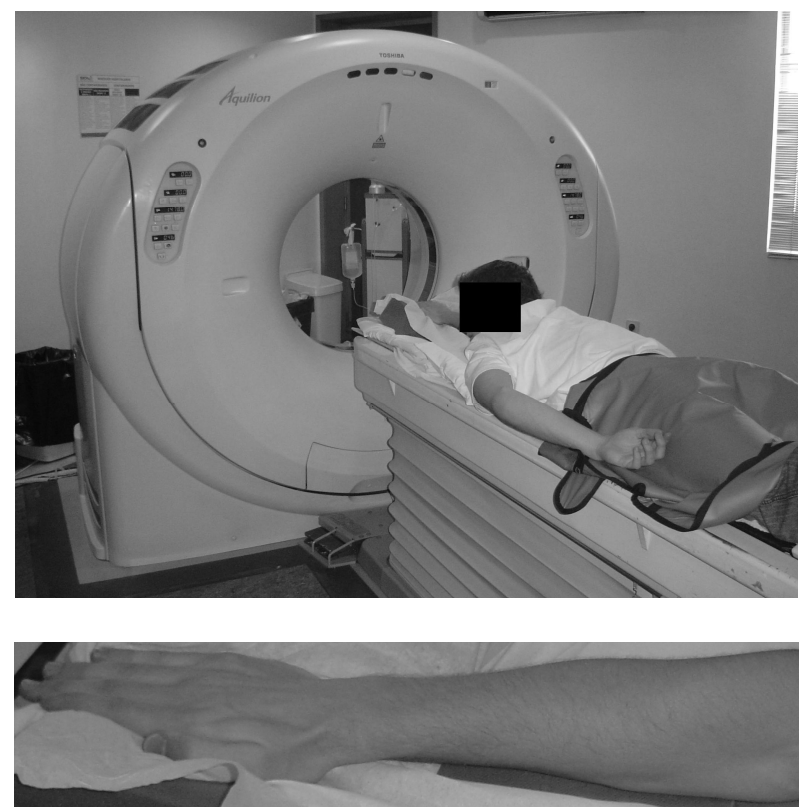

Figure 1 - Computer tomography scans protocol.

\section{Conversion into relevant coordinate input}

The transformation of values from the computer tomography scans into nodal coordinates in an appropriate coordinate system demands the use of image processing techniques. The image processing program used in this study was the Anatomics Pro ${ }^{\mathbb{B}}$. This program allowed obtaining the boundaries of the human segments, creating a three-dimensional reconstruction of the swimmer hand and forearm. This program uses computational functions, graphics functions and mouse functions.

At first, before processing and converting procedures the data was prepared, namely by observing the computer tomography data and erasing the non-relevant parts of the anatomical model. For example, surfaces supporting the subject were also scanned, reason why it had to be defined the relevant points and deleted the irrelevant ones. This step was also conducted using the software FreeForm Sensable ${ }^{\circledR}$. Finally, the data was converted into an IGES format (*.igs), that could be read by Gambit/Fluent ${ }^{\circledR}$ to define the finite elements approach through the three-dimensional surfaces.

\section{RESULTS AND DISCUSSION}

Figure 2 shows the hand and forearm model produced by the image processing techniques. It showed an almost perfect agreement with the true human segment. This technique could lead to overcome the difficulty to develop a true threedimensional model of a specific segment of the human body. 

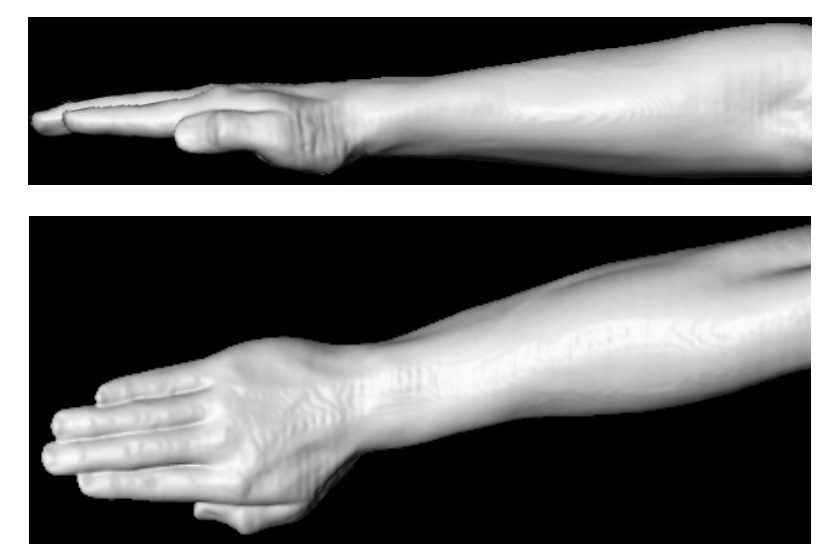

Figure 2 - Two different perspectives of the hand and forearm model produced by the image processing techniques.

One of the main limitations of the application of numerical techniques in swimming research is the quality of the models used to represent the human limbs. Indeed, there are few studies applying this computational tool in the analysis of human swimming propulsion and they used very simple representations of the human body (Bixler and Schloder, 1996; Silva et al., 2005; Gardano and Dabnichki, 2006; Rouboa et al., 2006). Despite the important contributes of those studies, the design and the use of a more realistic model is an essential feature to the development of Computational Fluid Dynamics in swimming analysis (Gardano and Dabnichki, 2006).

The generation of a three-dimensional model from the computer tomography scans of the human limbs has been used in other fields, such as in biomedical and engineering scopes (e.g. Tu et al., 1995; Aritan et al., 1997; Long et al., 1998). Prosthetic design and analysis, for instance, require a correct description of the limb geometry, allowing the output from the program to be used as a digital input for manufacturing prosthetics or limb models. Moreover, the magnetic resonance imaging techniques could allow clear separation of the skin, fat and muscle layers. Furthermore, the local compartment fat content can be estimated with a high level of accuracy (Aritan et al., 1997). These applications suggest that there is scope for some physiological applications such as finding muscle cross-sections or volumes. However, in this study, only the outer contour of the hand and forearm were considered. The generation of the mesh of cells to define the finite elements, allowing the analysis of the fluid flow takes place around those segments. The mesh of the whole domain was defined in Gambit Module, a mesh tool of the Fluent ${ }^{\circledR}$ software. The hand/forearm model was placed centred in the domain with larger dimensions in order to determine the undisturbed boundary conditions. The schematic design of the computational model is shown in Figure 3. The whole domain was meshed with a hybrid mesh composed of prisms and pyramids.

With this model, it was possible to export the generated mesh to the Fluent ${ }^{\circledR}$ processor, where the conditions suitable to be computed were defined. It was possible to compute the force values produced by the hand and forearm model through the integration of pressures on the hand/forearm surfaces (Fig. 4). Fluent software allows the analysis of the force components in the three directions of the space, with the model with different orientations, as it occurs in swimming.

However, one should note that simulation or modelling of water flow conditions around the hand and forearm when treated as one segment does not illustrate the complexity of propulsion generating process. It is widely known that propulsion generated by the upper extremity is a result of mutual displacement of each segment in three dimensions (Toussaint et al., 2002). 


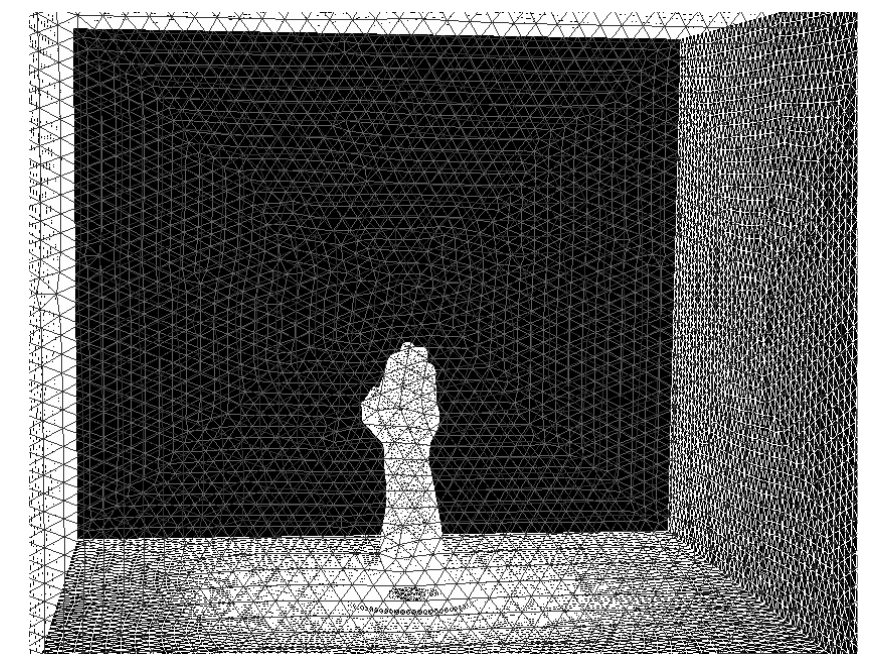

Figure 3 - Hand and forearm model inside the domain with three-dimensional mesh of cells.

Moreover, the upper arm can provide effective propulsion through most of the stroke (Gardano and Dabnichki, 2006). Thus, further research must consider these concerns and the movement at the wrist, elbow, and shoulder joints must be added in the modelling of the arm propulsion. The human model obtained with computer tomography scans can be used to evaluate accurately various aspects of unsteady motion, such as accelerations, decelerations, and multi-axis rotations of the propelling segments. This aim could be achieved by performing transient time-dependent numerical simulations including user-defined functions and moving meshes allowing simulating the swimmer's movements (Lyttle and Keys, 2006). Thus, the computer tomography scans allowed the creation of a complete and true digital anatomic model of a swimmer hand and forearm. This could help to improve the use of Computational Fluid Dynamics in swimming studies, decreasing the gap between the experimental and the computational data.

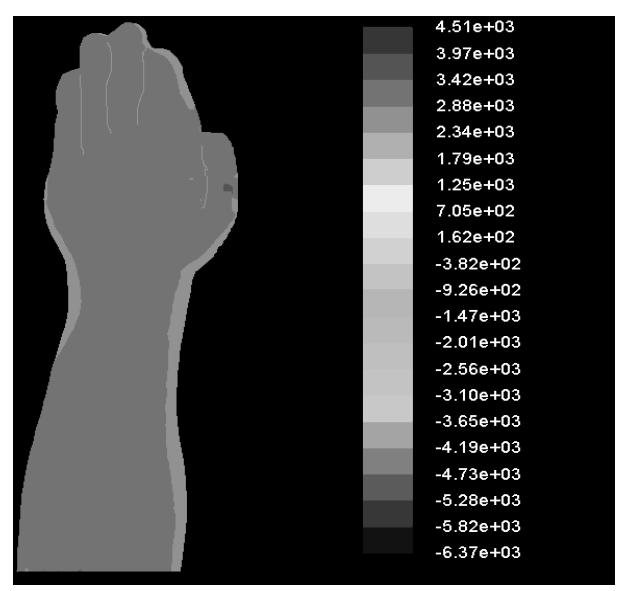

Figure 4 - Computational vision of the relative pressure contours on the hand/forearm surfaces.

\section{ACKNOWLEDGMENT}

This work was supported by the Portuguese Government by a grant of the Science and
Technology Foundation (SFRH/BD/25241/2005; POCI/DES/58872/2004). We would like to acknowledge the staff of the Radiology Department of Hospital de São João, from Porto, 
Portugal, and personally to the Department Director, Professor Isabel Ramos. We would also like to express our gratitude to the Med Mat Innovation Company, from Maia, Portugal, especially to the Professor José Domingos Santos and the Engineer Bruno Sá for their contributes.

\section{RESUMO}

O objetivo do presente estudo foi desenvolver um modelo digital tridimensional de uma mão e um antebraço humano para aplicar a Dinâmica Computacional de Fluidos ao estudo da propulsão em natação. Foram aplicados procedimentos computorizados de tomografia axial na mão e antebraço de um nadador Olímpico. Através de técnicas de processamento de imagem, os dados foram convertidos em coordenadas tridimensionais, que podem ser utilizadas em programas de simulação computacional. Através dos resultados encontrados, foi possível verificar uma semelhança quase perfeita entre o segmento humano e o modelo digital. Esta técnica pode ser utilizada como uma forma de ultrapassar as dificuldades em desenvolver um modelo digital tridimensional de um segmento específico do corpo humano. Complementarmente, pode ser bastante útil na melhoria da utilização da Dinâmica Computacional de Fluidos no Desporto, de uma forma geral, e, mais especificamente, nos estudos em natação, diminuindo a diferença entre a investigação experimental e a investigação computacional.

\section{REFERENCES}

Aritan, S.; Dabnichki, P.; Bartlett, R. (1997), Program for generation of three-dimensional finite element mesh form magnetic resonance imaging scans of human limbs. Medical Engineering and Physics, 19 (8), 681-689.
Bixler, B.S.; Schloder, M. (1996), Computational fluid dynamics: an analytical tool for the 21 st century swimming scientist. Journal of Swimming Research, 11, 4-22.

Candalai, R.S.; Reddy, N.P. (1992), Stress distribution in a physical buttock model: effect of simulated bone geometry. Journal of Biomechanics, 15, 493-504.

Gardano, P.; Dabnichki, P. (2006), On hydrodynamics of drag and lift of the human arm. Journal of Biomechanics, 39, 2767-2773.

Lyttle, A.; Keys, M. (2006), The application of computational fluid dynamics for technique prescription in underwater kicking. Portuguese Journal of Sport Sciences, 6 (Suppl. 2), 233-235.

Long, Q.; Xu, X.Y.; Collins, M.W.; Griffith, T.M.; Bourne, M. (1998), The combination of magnetic resonance angiography and computational fluid dynamics: a critical review. Critical Reviews in Biomedical Engineering, 26 (4), 227-274.

Rouboa, A.; Silva, A.; Leal, L.; Rocha, J.; Alves, F. (2006), The effect of swimmer's hand/forearm acceleration on propulsive forces generation using Computational Fluid Dynamics. Journal of Biomechanics, 39 (7), 1239-1248.

Silva, A.; Rouboa, A.; Leal, L.; Rocha, J.; Alves, F.; Moreira, A.; Reis, V.; Vilas-Boas, J.P. (2005), Measurement of swimmer's hand/forearm propulsive forces generation using computational fluid dynamics. Portuguese Journal of Sport Sciences, 5 (3), 288-297.

Toussaint, H.M.; Van Den Berg, C.; Beek, W.J. (2002), "Pumped-up propulsion" during front crawl swimming. Medicine and Science in Sports and Exercise, 34 (2), 314-319.

Tu, H.K.; Matheny, A.; Goldgof, D.B.; Bunke, H. (1995), Left ventricular boundary detection from spatio-temporal volumetric computer tomography images. Computerized Medical Imaging and Graphics, 19 (1), 27-46.
Received: May 24, 2008; Revised: November 07, 2008; Accepted: October 05, 2009. 